\title{
Uniformly best wavenumber approximations by spatial central difference operators: An initial investigation
}

Viktor Linders and Jan Nordström

\begin{abstract}
A characterisation theorem for best uniform wavenumber approximations by central difference schemes is presented. A central difference stencil is derived based on the theorem and is compared with dispersion relation preserving schemes and with classical central differences for a relevant test problem.
\end{abstract}

\section{Introduction}

Modelling wave propagation over sizeable intervals using finite differences is a common problem in fields ranging from aeroacoustics to seismology. For high frequency problems the numerical error may over time be dominated by inaccurate approximations of the dispersion relation, leading to errors in phase and group velocity, unless the spatial increment, $\Delta x$ is very small.

A remedy, presented in [1] for central differences, is to perturb the classical schemes by an extra parameter but decreasing the formal accuracy. The new parameter is used to minimise the dispersion error in the $L^{2}[0, \pi / 2]$ norm. Such schemes are known as Dispersion Relation Preserving (DRP). For other approaches based on similar ideas, see e.g. [2, 3, 4].

The DRP approach is disadvantageous in that it provides no means of obtaining wavenumber-specific error bounds. For problems involving a range of wavenumbers it is more convenient to minimise the dispersion error in the $L^{\infty}$-norm. In this paper we present a characterisation theorem for best uniform wavenumber approxima-

Viktor Linders

Linköping University, Department of Mathematics, Computational Mathematics, SE-581 83

Linköping, Sweden, e-mail: viktor.linders@liu.se

Jan Nordström

Linköping University, Department of Mathematics, Computational Mathematics, SE-581 83

Linköping, Sweden, e-mail: jan.nordstromeliu.se 
tions. New central difference schemes are derived and compared with their classical and DRP counterparts.

\section{Central difference schemes}

We begin by demonstrating why classical central differences are suboptimal for wavenumber approximation. Consider a central difference stencil of order $2 p$,

$$
\left(u_{x}\right)_{j}=\frac{1}{\Delta x} \sum_{k=1}^{p} c_{k}^{(p)}\left(u_{j+k}-u_{j-k}\right)+\mathscr{O}\left(\Delta x^{2 p}\right) .
$$

The numerical wavenumber of this scheme is (see e.g. [1])

$$
\bar{\xi}_{c}=2 \sum_{k=1}^{p} c_{k}^{(p)} \sin (k \xi)
$$

where $\xi=\Delta x \kappa$ and $\kappa$ is the exact wavenumber of the propagating solution. Here we let $\xi \in\left[0, \xi_{\text {max }}\right] \subseteq[0, \pi]$.

A Taylor expansion reveals that to obtain desired accuracy, $c_{k}^{(p)}$ must satisfy

$$
\left(\begin{array}{cccc}
1 & 2 & \ldots & p \\
1 & 2^{3} & \ldots & p^{3} \\
\vdots & \vdots & & \vdots \\
1 & 2^{2 p-1} & \ldots & p^{2 p-1}
\end{array}\right)\left(\begin{array}{c}
c_{1}^{(p)} \\
c_{2}^{(p)} \\
\vdots \\
c_{p}^{(p)}
\end{array}\right)=\left(\begin{array}{c}
\frac{1}{2} \\
0 \\
\vdots \\
0
\end{array}\right) .
$$

The following observation is useful. For the proof, see [5].

Lemma 1. Consider the function

$$
f_{p}(x)=1-2 \sum_{k=1}^{p} c_{k}^{(p)} k T_{k}(x)
$$

where $c_{k}^{(p)}$ satisfies (2) and $T_{k}(x)$ is the $k^{\text {th }}$ order Chebyshev polynomial of the first kind, uniquely defined through the relation $T_{k}(\cos (\phi))=\cos (k \phi)$. Then

$$
f_{p}(x)=d_{p}(1-x)^{p}
$$

for some $d_{p}$ that depends exclusively on $p$.

Theorem 1. Classical central difference stencils underestimate the speed of propagating solutions.

Proof. Let

$$
E_{c}(\xi) \equiv \xi-\bar{\xi}_{c}=\xi-2 \sum_{k=1}^{p} c_{k}^{(p)} \sin (k \xi)
$$


be the error function associated with a classical central difference stencil of order $\mathscr{O}\left(\Delta x^{2 p}\right)$. Note from the definition of $f_{p}$ and the Chebyshev polynomials that

$$
\frac{\mathrm{d} E_{c}}{\mathrm{~d} \xi}=f_{p}(\cos (\xi))
$$

It follows that $\frac{d E_{c}}{\mathrm{~d} \xi}=0$ only when $\cos (\xi)=1$. It is thus clear that $E_{c}(\xi)$ has an inflection point at $\xi=0$ and no other extrema in the domain of interest. Consequently $E_{c}(\xi)$ is monotonic and since $E_{c}(0)=0$ and $E_{c}(\pi)=\pi$ it is also increasing. We thus have $\bar{\xi}_{c} \leq \xi$ with equality only at $\xi=0$. Therefore the classical central difference stencils underestimate the analytic wavenumber. It follows now that the relative error in the phase speed is

$$
\frac{\bar{v}_{p}-v_{p}}{v_{p}}=\frac{\bar{\xi}_{c}}{\xi}-1 \leq 0
$$

and so the phase speed is underestimated.

Let us now, like for DRP schemes, perturb the stencil by adding an additional coefficient, $a_{p+1}$ without increasing the accuracy. The coefficients of the new stencil must solve the system (2) for each $a_{p+1}$, though this system is now underdetermined. Calling the coefficients of the new system $a_{k}, k=1, \ldots, p+1$, we must have a linear dependence of the first $p$ coefficients on the added parameter, $a_{p+1}$. We write $a_{k}=c_{k}^{(p)}+c_{k}^{\prime} a_{p+1}, k=1, \ldots, p$.

In view of (3) the numerical wavenumber of the perturbed stencil is

$$
\bar{\xi}=2 \sum_{k=1}^{p+1} a_{k} \sin (k \xi), \quad 0 \leq \xi \leq \xi_{\max } \leq \pi
$$

Let us define the error function of this scheme as

$$
E(\xi) \equiv \xi-\bar{\xi}=\xi-2 \sum_{k=1}^{p+1} a_{k} \sin (k \xi)
$$

Our goal is to choose $a_{p+1}$ such as to minimise the magnitude of any extrema of $E(\xi)$. In order to do so we will extend Lemma 1 to the perturbed stencil. For a detailed proof, see [5].

Lemma 2. Let

$$
g_{p}(x)=1-2 \sum_{k=1}^{p+1} a_{k} k T_{k}(x)
$$

where $a_{k}=c_{k}^{(p)}+c_{k}^{\prime} a_{p+1}$ are the coefficients of the perturbed central difference stencil as defined previously, and $T_{k}(x)$ is the $k^{\text {th }}$ order Chebushev polynomial. Then

$$
g_{p}(x)=(1-x)^{p}\left[(1-x) \frac{d_{p+1}}{c_{p+1}^{p+1}} a_{p+1}+d_{p}\left(1-\frac{a_{p+1}}{c_{p+1}^{p+1}}\right)\right]
$$


where $d_{p}$ and $c_{k}^{(p)}$ are defined as before.

Corollary 1. $E(\xi)$ has at most one extremum in the open interval $\left(0, \xi_{\text {max }}\right]$ and it is located at

$$
\xi=\xi_{r}=\arccos \left(1-\frac{d_{p}}{d_{p+1}}\left[1-\frac{c_{p+1}^{p+1}}{a_{p+1}}\right]\right) .
$$

For good approximations $\xi_{r}$ is a minimum. This occurs only when $a_{p+1}$ and $c_{p+1}^{p+1}$ have the same sign and $\left|a_{p+1}\right| \geq\left|c_{p+1}^{p+1}\right|$, where equality holds only for classical stencils.

Again, for the proof we refer to [5]. From the above corollary we conclude that we can have $|E(\xi)|=\|E\|_{\infty}$ only at two possible points, namely at $\xi_{r}$ or $\xi_{\text {max }}$, i.e.

$$
\|E\|_{\infty}=\max \left\{\left|E\left(\xi_{\max }\right)\right|,\left|E\left(\xi_{r}\right)\right|\right\} .
$$

Of course $E\left(\xi_{r}\right)$ and $E\left(\xi_{\text {max }}\right)$ depend on how we choose $a_{p+1}$ and in view of (5) it is of interest to investigate this dependency. Our goal is to find the choice of $a_{p+1}$ that minimises (5). In fact we have

Theorem 2. Consider a $2 p+3$ point central difference scheme of order $\mathscr{O}\left(\Delta x^{2 p}\right)$ with numerical wavenumber $\bar{\xi}$ defined as in (3), and a corresponding error function $E(\xi)=\xi-\bar{\xi}$. The stencil that uniformly minimises the error function, i.e. $\min _{\bar{\xi}}\|E\|_{\infty}=\min _{a_{p+1} \in \mathbb{R}}\|\xi-\bar{\xi}\|_{\infty}$, is uniquely characterised by the property

$$
E\left(\xi_{r}\right)+E\left(\xi_{\max }\right)=0 .
$$

The proof is found in [5] where it is also demonstrated how this result generalises to an arbitrary number of free parameters, $a_{p+1}, \ldots, a_{p+n}$.

\section{A numerical example}

For a given $\xi_{\text {max }}$ solving (6) is a simple matter. Even for general $\xi_{\text {max }}$ good estimates may be found by replacing $E\left(\xi_{r}\right)$ by a suitable polynomial approximation. For the case $p=1$ this is shown in Fig. 1 for $\xi_{\max } \in[0, \pi / 2]$. Here $e=-\min _{\bar{\xi}}\|E\|$.

To illustrate the strength of Theorem 2 we consider a profoundly polychromatic solution to the advection equation over a periodic domain:

$$
\begin{aligned}
& u_{t}+u_{x}=0, \quad 0 \leq x \leq 3, \quad t \geq 0 \\
& u(x, 0)=\exp \left(-3200(x-1 / 2)^{2}\right) .
\end{aligned}
$$

This pulse is narrow and its Fourier transform is wide resulting in a significant contribution from a broad range of wavenumbers. The dominating wavenumber is 


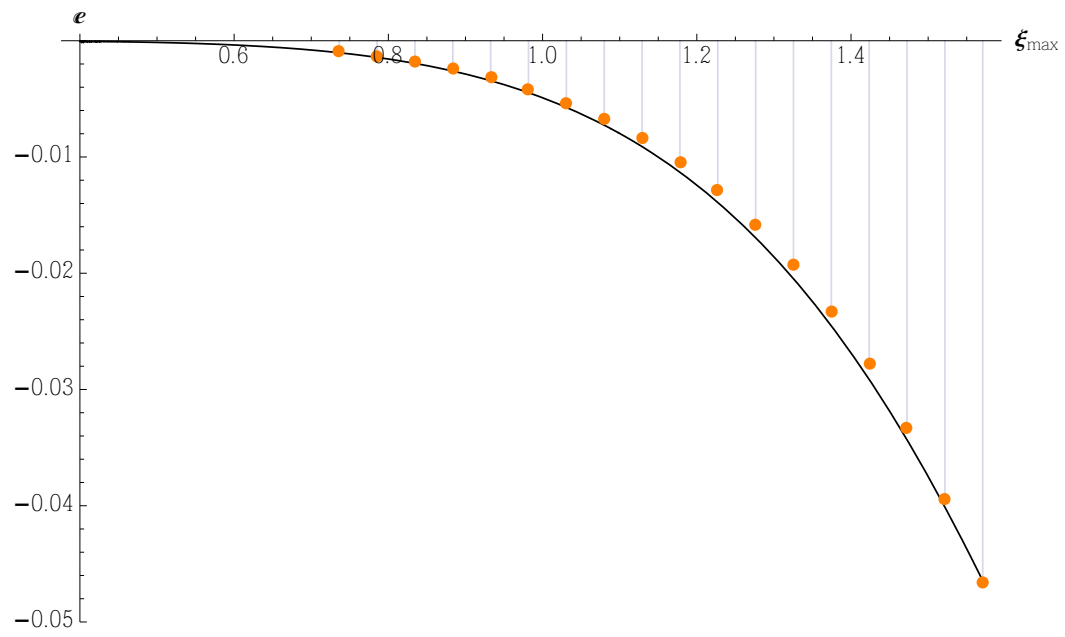

Fig. 1: Error of best uniform wavenumber approximation for given $\xi_{\max } \in[0, \pi / 2]$ (orange dots) and general solution using third degree polynomial approximation (black line).

$\kappa=0$. Contributions from larger wavenumbers decay exponentially but slowly. It makes sense to use a scheme that accurately approximates the dispersion relation near $\xi=\kappa \Delta x=0$ and for some suitably chosen region of larger wavenumbers.

From Fig. 1 we see that if we choose $\xi_{\max }=\pi / 4 \approx 0.785$ we will have an error in the dispersion relation of around $10^{-3}$. Solving (6) gives the scheme

$$
a_{1}=0.683345936919182, \quad a_{2}=-0.091672968459591 .
$$

In Fig. 2 the dispersion error of the new scheme is plotted as a function of $\xi$. The approximation starts to deviate from the exact result for $\xi \geq \xi_{\max }$. The errors of a fourth order classical central difference scheme and of a five-point DRP scheme [1] are also included. As expected from Theorem 1 the classical stencil underestimates the dispersion relation, seen by the positive sign of the error. For the shown range of $\xi$ it seems that the DRP scheme overestimates the dispersion relation whereas our new scheme stays within tight error bounds.

We set $\Delta x=1 / 120$ and integrate in time using the classical fourth order RungeKutta scheme with time step $\Delta t=10^{-3}$ so the contribution from the temporal discretisation is small. The exact and numerical solutions are shown in Fig. 3 (top) together with the error as a function of time (bottom). All numerical solutions quickly disperse into a train of pulses of decaying amplitude trailing behind the main peak. As expected from Fig. 2, the DRP scheme overestimates the speed of some pulses. Our new scheme does this as well but to a much reduced extent. The smaller pulse train behind the DRP solution with respect to the new scheme may be attributed to a better approximation for very high wavenumbers. However, since the contribution of these wavenumbers are comparably small, the resulting error remains larger for the DRP scheme as compared with the classical and the new stencil. 


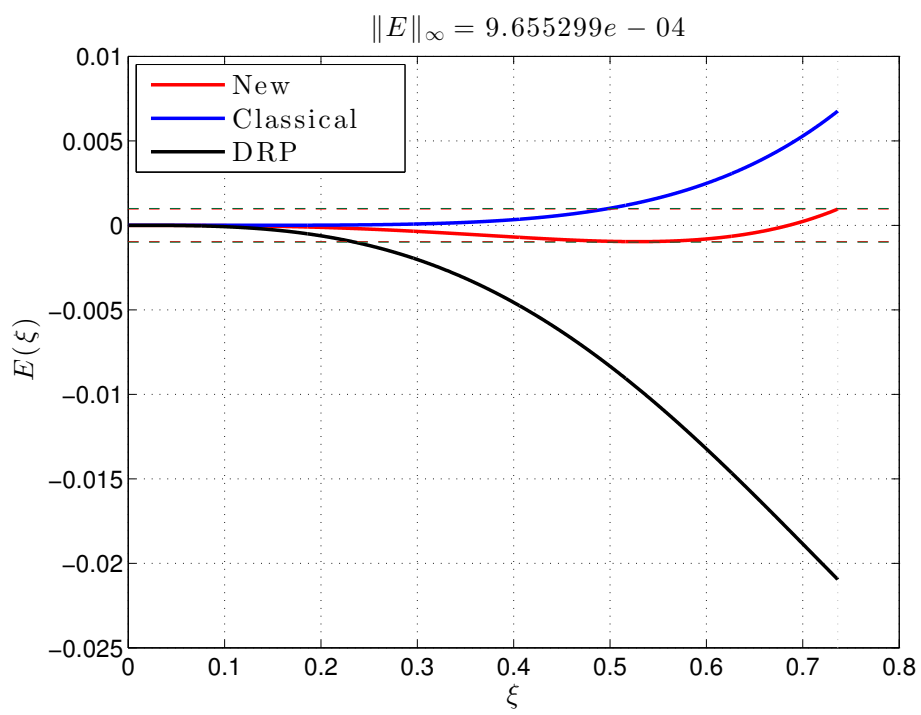

Fig. 2: Dispersion error for the new scheme, the classical $4^{\text {th }}$ order stencil, and a five-point DRP scheme.

\section{Extension to multiple dimensions}

Extending the new stencil to multiple dimensions is in principle straight forward. As an example, for the advection problem in 2D we may, after discretising in space, write

$$
\mathbf{v}_{t}+\left(D_{x} \otimes I_{y}\right) \mathbf{v}+\left(I_{x} \otimes D_{y}\right) \mathbf{v}=0
$$

where $\mathbf{v}$ is a grid vector approximating the true solution, $D_{x, y}$ are periodic operators containing the central difference stencil and operating on a given cartesian grid in the $x$ and $y$ direction respectively. Here $I_{x, y}$ are identity matrices of appropriate dimensions and $\otimes$ denotes the Kronecker product.

For this situation, the solution propagates at an angle $\theta$ with respect to the $x$ axis. It should be noted that the stencils presented here are optimal for the onedimensional problem, that is for the cases when $\theta=n \pi / 4, n=0,1,2,3$. For any other angle the stencils will be suboptimal since the numerical dispersion relation depends on the direction of propagation. In other words, these stencils may be sensitive to numerical anisotropy. For a comprehensive overview of methods that handles this issue, see e.g. [6]. At present we shall not consider this phenomenon further. 

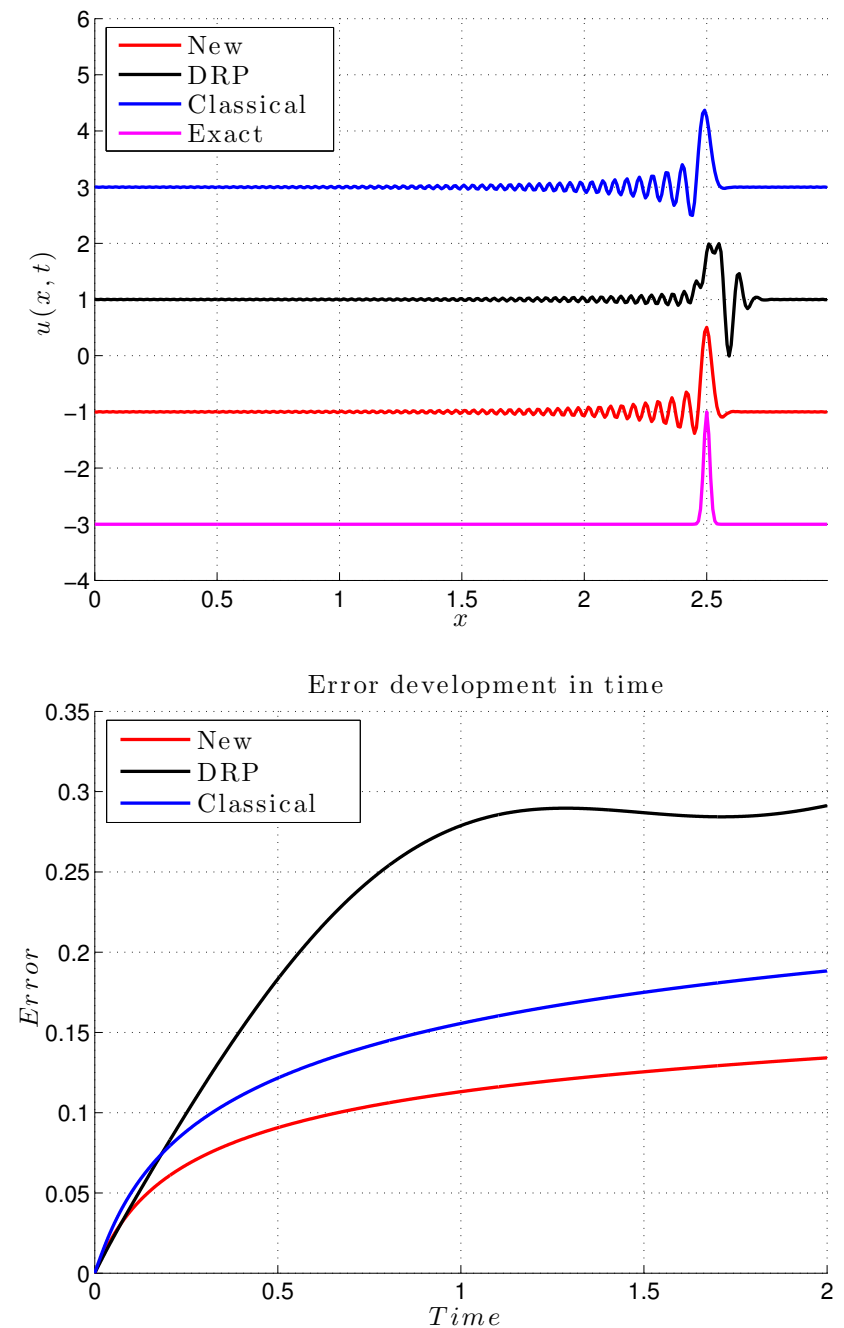

Fig. 3: (Top) Exact solution and numerical approximations after 2000 time steps. (Bottom) Corresponding errors.

\section{Conclusion}

We have proved a characterisation theorem for best uniform wavenumber approximations by central difference stencils with one free parameter. The best approximation is unique and may be easily obtained numerically for a given range of wavenumbers. This allows for accurate approximations of problems of high frequency waves, or multi-frequency solutions, with a relatively coarse spatial mesh. 


\section{References}

1. C.K.W. Tam, J.C. Web, Dispersion-Relation-Preserving Finite Difference Schemes for Computational Acoustics, J. Comput. Phys. 107, 262-281 (1993)

2. D.W. Zing, H. Lomax, H. Jurgens, High-accuracy finite-difference schemes for linear wave propagation, SIAM J. Sci. Compute. 17, 328-346 (1996)

3. D.W. Zingg, H. Lomax, H. Jurgens, An optimized finite-difference scheme for wave propagation problems, AIAA paper 93(0459) (1993)

4. C. Bogey, C. Bailly, A family of low dispersive and low dissipative explicit schemes for flow and noise computations, J. Compute. Phys. 194, 194-214 (2004)

5. V. Linders, J. Nordström, Uniformly best wavenumber approximations by spatial central difference operators, LiTH-MAT-R, 2014:17, 2014, Department of Mathematics, Linköping University

6. A. Sescu, Numerical anisotropy in finite differencing, Advances in Difference Equations 2015:9 (2015), Mississippi State University 